\title{
SDE based Generalized Innovation Diffusion Modeling
}

\author{
Shakshi Singhal, Adarsh Anand*, Ompal Singh \\ Department of Operational Research \\ University of Delhi, Delhi-110007, India \\ *Corresponding author: adarsh.anand86@gmail.com
}

(Received May 22, 2018; Accepted February 21, 2019)

\begin{abstract}
Diffusion models are rigorously implemented in marketing research to predict the actual trend of innovations over time. These models can be classified in terms of deterministic and stochastic behavior. Deterministic models ignore the randomness in the adoption rate of an innovation that occurs due to environmental and internal system disturbances. Therefore, in the present research, a generalized stochastic diffusion model using Itô's process is proposed that jointly study the product awareness and eventual adoption of an innovation. Convolution function is applied to integrate these two processes. In addition, different probability distributions are employed, which are relevant for describing the product awareness and adoption processes. Non-linear regression is further carried out to validate the proposed models and parameters are estimated based on the actual sales data from Smartphone and automobile industries. The forecasting results indicate that the proposed models perform empirically better than the already established diffusion models.
\end{abstract}

Keywords- Awareness, Convolution, Itô's integral, Stochastic differential equation, Technology diffusion.

\section{Introduction}

The Smartphone and automotive industries are the two most revolutionized and dominant industry in the globe. The literature survey has demonstrated that the advancement of Smartphone and hightech automobiles have transformed the entire picture of the market and has created enormous business opportunities for the enterprises. Considering the fast-evolving technologies, many studies have been conducted to estimate the growth pattern of these high-tech products. The widespread acceptance of Smartphone and automobile products provides opportunities and challenges for the academicians and practitioners to study how to model the adoption behavior and the diffusion process for new products and technologies. The study of adoption behavior is obtained from notions and theories of individual decision-making (Aronov and Zazhigalkin, 2017). Based on the individual's propensity to adopt, they are commonly classified as 'innovators', 'early adopters', etc (Rogers, 2003). The product diffusion describes and foresees the time of the adoption of new technological products among the potential market. It is analogous to an epidemic process wherein users persuade non-adopters by interpersonal communication leading to eventual adoption. As the success of technological products is influenced by its diffusion pattern, the modeling and predicting of the dissemination of new products have gained considerable academic attention (Meade and Islam, 2006).

The purpose of diffusion models is to produce predictions about the timing of adoption of new technological products by individuals to better interpret the attitude and buying behavior of users. This will facilitate the manufacturers in decision-making pertaining to production, finance, and marketing. The most prominent and highly cited diffusion model in marketing science was suggested by Bass (1969). Since the introduction of the Bass model, it has been the main impetus in analyzing the dispersion of innovations in the marketplace (Anand et al., 2018). The Bass model is guided by the assumption that new products are propagated through two kinds of persuasive 
International Journal of Mathematical, Engineering and Management Sciences

Vol. 4, No. 3, 697-707, 2019

https://dx.doi.org/10.33889/IJMEMS.2019.4.3-055

communication channels, specifically, mass-media and interpersonal communication. Bass (1969) termed the prospective buyers as innovators if they adopt the innovation under the influence of mass communication and as imitators if their purchasing decision is swayed by the word-of-mouth effect. The Bass model is broadly employed in new product forecasting, business model diffusion, and technological diffusion due to its easy mathematical framework and the precise economic implications of the parameters (Wang et al., 2017; Singhal et al., 2019).

However, the conventional Bass (1969) model is established on various assumptions that restrict the model pragmatic prediction capability. For instance, the Bass model does not take into account the effect of marketing-mix variables such as pricing, advertising outlay onto the adoption process. In addition, the classical model assumes that the adoption process occurs instantaneously, i.e. the prospective individuals will buy the product as soon as they get aware of the innovation. Nevertheless, there exists a considerable time lag between the propagation of knowledge about the new technology among the potential market and its final adoption. Rogers (2003), in his research on innovation diffusion process, claims that adopters go through several stages before confirming their purchasing decision. According to his theory, adoption process occurs in five stages: knowledge (information about the new technology is circulated among the probable people), persuasion (people seek further knowledge to form an opinion for the new product), evaluation (prospective buyer makes a decision either to buy or reject the product), implementation (the individual put the new technology into use for trial), confirmation (the individual eventually purchase the innovation if he/she is satisfied from its trial run). Consequently, it is prudent to observe the effect from different stages on the diffusion dynamics. In the present study, the adoption process of automobile and Smartphone products are evaluated using the two-stage structure: knowledge or awareness stage and confirmation or eventual adoption stage.

Another limitation of the Bass model is that it assumed the uncertainty on the diffusion path to be constant over time. However, in a practical scenario, the underlying techniques of product diffusion are stochastic. For instance, the underlying process whether an individual connects with the masscommunication service and purchase the innovation under its influence is uncertain. Similarly, the adoption because of the persuasion by the existing users through word-of-mouth communication is also theoretical. Therefore, for the rational depiction of the diffusion process, the uncertainty should be considered in the modeling framework. Although the characteristic of the diffusion process is inherently stochastic, the more emphasis is given to the deterministic modeling structure. Because the differential equation involving nonlinear stochastic modeling framework is intricate and complex to solve, one usually assumes the process deterministic and certain. The purpose of the current research is to overcome the limitation of the classical diffusion models by developing a two-stage diffusion model for technological innovations while incorporating randomness in the adoption rate. A stochastic differential equation based mathematical model with time delay structure is formulated that measures the awareness of information and the eventual adoption of the new technology. The remaining article is outlined into following sections. Section 2 summarizes the literature review on innovation diffusion modeling. The problem description and proposed methodology is described in Section 3. Subsequently, an empirical analysis is carried out in Section 4 to evaluate the forecasting and estimation capability of the proposed modeling framework. Finally, conclusion is provided in Section 5.

\section{Literature Review}

In marketing and technology management literature, a good deal of diffusion models has been proposed to illustrate the degree of dissemination of new technologies and to predict their expected 
International Journal of Mathematical, Engineering and Management Sciences

Vol. 4, No. 3, 697-707, 2019

https://dx.doi.org/10.33889/IJMEMS.2019.4.3-055

demand. Bass (1969) introduced the initial diffusion model with an objective of depicting the dispersion of consumer durable innovations. According to Bass (1969), the cumulative sale behavior of new products is structured by an S-shaped curve. Although the Bass model has yielded an effective framework for interpreting the appropriate diffusion process of new technological products, it is based on several assumptions. Therefore, in the past, many researchers have attempted to extend the Bass model to assimilate other aspects of the diffusion process (Meade and Islam, 2006; Chandrasekaran and Tellis, 2007).

An important factor added in the Bass model was the multi-stage diffusion process. It is practical to assume that before purchasing an innovation, individual first gather knowledge about its existence and then make a buying decision. Based on this supposition, Kalish (1985) presented a diffusion model with a two-stage adoption process: awareness and adoption. In the awareness stage, probable buyers are exposed to the existence of the innovation and in the adoption stage, aware potential market process information regarding the new product, which they have gathered and then carries out a purchasing decision. Lal et al. (1988) have also modeled the adoption process with a time lag function between the knowledge stage and eventual adoption stage. In 2010, Landsman and Givon developed a two-stage diffusion process for service innovation. Later, several authors have made an effort to model a new product diffusion process with stage structure (Wang et al., 2006, Fanelli and Maddalena, 2012; Anand et al. 2014, 2016; Agarwal et al. 2017). However, the limitation of these studies is that they assumed the diffusion process to be deterministic and have ignored the uncertainty in the adoption behaviour.

The variation in the factors affecting the diffusion process, whether small or wide, can cause randomness in customer's purchasing decision (Anand and Bansal, 2016). The ambiguity in the consumer's buying assessment can tremendously influence manufacturers, distributors and retailers ordering and reserve keeping policies. Therefore, the stochastic examination of the diffusion process by including the rapidly changing environmental and internal attributes is necessary for generating a dependable future prediction. In innovation diffusion modeling, Eliashberg and Chatterjee (1986) were the first to introduce the stochastic behavior by allowing the diffusion parameters to follow the stationary stochastic process. Giovanis and Skiadas (1999) further proposed the stochastic diffusion model for electricity consumption in Greece and in the United States. They formulated the logistic growth model by using the Itô's stochastic differential equation (SDE). Later, Gutiérrez et al. (2005) have developed the Gompertz stochastic diffusion model for natural gas consumption in Spain. In the past, authors have also extended the Bass diffusion theory for stochastic consideration. Boswijk and Franses (2005) have added the stochastic error term in the Bass diffusion process to produce reliable parameter estimates. Singh et al. (2012) have developed the stochastic diffusion process by incorporating randomness in the adoption rate. Negahban and Smith (2014) have used the Bass diffusion model to estimate the demand for new products. They have explored the uncertainty in the production function and its impact on the product's introduction time and sales plan by using stochastic supply constraint. Quite recently, Anand et al. (2018) have also extended the Bass diffusion model by incorporating dynamic market size and irregular fluctuation in the adoption rate.

Despite the importance of stochastic consideration in the diffusion behavior of new products, only few studies have been conducted to understand the structural and conceptual assumptions underlying diffusion models in the stochastic environment. Therefore, the purpose of the present research is to model the diffusion behavior mathematically with stage structure by considering random fluctuation in the adoption process. A unified approach is used to model the adoption 
International Journal of Mathematical, Engineering and Management Sciences

Vol. 4, No. 3, 697-707, 2019

https://dx.doi.org/10.33889/IJMEMS.2019.4.3-055

process under two stages: awareness of information and eventual adoption. A unification scheme is an intuitive analysis for the study of mathematical models that helps in combining several theories into one structure. The two stages of the adoption process are integrated into the diffusion process using the convolution function. Convolution is a mathematical operation on two different functions to generate a new function having the characteristics of both the input functions. The developed stochastic differential equation based diffusion model is further solved using the Itô process. The instances from automotive and Smartphone industries are taken to investigate the practical applicability of the proposed model.

\section{Problem Description and Mathematical Model \\ 3.1 Notations}

$\begin{array}{ll}m & \text { Fixed and definite market size of the technology innovation } \\ N(t) & \text { Cumulative demand function } \\ F_{a w}(t) & \text { Distribution function for awareness of information process } \\ F_{a d}(t) & \text { Distribution function for eventual adoption process } \\ b & \text { Adoption parameter } \\ \beta & \text { Learning parameter of logistic distribution function } \\ \mu & \text { Mean of normal distribution } \\ s & \text { Standard deviation of normal distribution } \\ * & \text { Convolution operator } \\ \otimes & \text { Stieltjes convolution operator }\end{array}$

\subsection{Model}

When randomness and irregular functions are allowed in any of the differential equation coefficients, then mathematical modeling represents a diffusion process in a more realistic form. The ordinary differential equations with randomness are often known as Langevin equation. These equations explain the Brownian motion or the Wiener process that are solved through either Itô stochastic calculus or Stratonovich stochastic calculus (Øksendal, 2003).

Using the unified approach, the ordinary differential equation for the demand function with single stage structure can be given as:

$$
\frac{d N(t)}{d t}=h(t)(m-N(t))
$$

where $h(t)=\frac{f(t)}{1-F(t)}$ denotes the hazard rate or the rate of adoption at any time ' $t$ ' and $(m-N(t))$ represents the untapped market potential who are yet to make the purchase. In order to construct a stochastic based diffusion model, the behavior of adoption rate is taken uncertain affected by the random environmental and internal factors. Thus, adoption rate is considered stochastic by adding a random term, i.e.

$h(t)=\frac{f(t)}{1-F(t)}+{ }^{\prime}$ noise' 
International Journal of Mathematical, Engineering and Management Sciences

Vol. 4, No. 3, 697-707, 2019

https://dx.doi.org/10.33889/IJMEMS.2019.4.3-055

where 'noise' term represents the fluctuation in the rate of adoption. It has been established that the exact behavior of noise is not deterministic and only its probabilistic behavior is known. By utilizing the principal of Weiner process, the 'noise' term can be expressed as (Øksendal, 2003):

$h(t)=\frac{f(t)}{1-F(t)}+\sigma \omega(t)$

where $\omega(t)$ is a random variable recognized as a standard Gaussian white noise; $\sigma$ is a positive constant, which describes the degree of the irregular fluctuation. By substituting the expression of random adoption rate in equation (1), the corresponding stochastic differential equation becomes:

$\frac{d N(t)}{d t}=\frac{f(t)}{1-F(t)}(m-N(t))+\sigma \omega(t)(m-N(t))$

The variable $\omega(t)$ is evaluated as a derivative of the one-dimensional Weiner process:

$\frac{d W(t)}{d t}=\omega(t)$

The Wiener process $W_{t}$ is a kind of continuous stochastic process possessing following properties:

(i) $W_{0}=0$

(ii) $W_{t}$ has independent increments for every $t>0$.

(iii) $W_{t}$ has Gaussian increments: $W_{t+u}-W_{t}$ follows normal distribution with mean 0 and variance $u, W(t+u)-W(t) \sim N(0, u)$

(iv) $W_{t}$ has continuous paths with probability 1.

By using the expression of equation (5) and using the Itô stochastic calculus, the stochastic differential equation (4) can be extended to the following Itô type equation: (Singh et al., 2012; Anand et al., 2018):

$d N(t)=\left(\frac{f(t)}{1-F(t)}-\frac{1}{2} \sigma^{2}\right)(m-N(t)) d t+\sigma(m-N(t)) d W(t)$

By using the Itô formula, the equation (6) can be solved under the condition $N(0)=0$ to form a cumulative demand function:

$N(t)=m\left[1-(1-F(t)) e^{-\sigma W(t)}\right]$

If $F(t)$ represents the adoption distribution function then the expected number of adopters can be written as: 
International Journal of Mathematical, Engineering and Management Sciences

Vol. 4, No. 3, 697-707, 2019

https://dx.doi.org/10.33889/IJMEMS.2019.4.3-055

$\bar{N}(t)=E[N(t)]=m\left[1-(1-F(t)) e^{\sigma^{2} t / 2}\right]$

Equation (8) depicts the mean value function of demand of new products under a single-stage structure.

Now, in order to describe a stochastic diffusion model with time delay function, the adoption process is structured using a two-stage process. The first stage, know as awareness of information stage is concerned with process that illustrates dissemination of product knowledge among the target potential market. The target market seeks knowledge about the product and transforms into a potential buyer who are aware of its existence. After processing the information that potential individuals have received about the innovation, they make the buying decision in the second (eventual adoption) stage. A convolution theory is applied in the hazard rate function to model the two-stage process in the diffusion paradigm. Thus, the generalized two-stage stochastic diffusion model can be expressed as:

$\frac{d N(t)}{d t}=\left(\frac{\left(f_{a w} * f_{a d}\right)(\mathrm{t})}{\left[1-\left(F_{a w} \otimes F_{a d}\right)(t)\right]}+\sigma \omega(t)\right)(m-N(t))$

where $\frac{\left(f_{a w} * f_{a d}\right)(\mathrm{t})}{\left[1-\left(F_{a w} \otimes F_{a d}\right)(t)\right]}$ is the static awareness - adoption rate at any time ' $t$ '. Equation (9) can be further transformed into stochastic differential equation of an Itô type:

$d N(t)=\left(\frac{\left(f_{a w} * f_{a d}\right)(\mathrm{t})}{\left[1-\left(F_{a w} \otimes F_{a d}\right)(t)\right]}-\frac{\sigma^{2}}{2}\right)(m-N(t)) d t+\sigma(m-N(t)) d W(t)$

By applying Itô formula and initial condition $N(0)=0$ to equation (10), the cumulative demand function is formed as:

$N(t)=m\left[1-\left(1-\left(F_{a w} \otimes F_{a d}\right)(t)\right) e^{-\sigma W(t)}\right]$

where $\left(F_{a w} \otimes F_{a d}\right)(t)=\int_{0}^{t} F_{a d}(t-u) d F_{a w}(u)=\int_{0}^{t} F_{a w}(t-u) d F_{a d}(u)$. Now, by taking the expectation of equation (11), the mean value function becomes:

$\bar{N}(t)=m\left[1-\left(1-\left(F_{a w} \otimes F_{a d}\right)(t)\right) e^{\sigma^{2} t / 2}\right]$

By applying different distribution function for describing the product, awareness of information and eventual adoption process, different stochastic innovation diffusion models (SIDM) have been derived. It is assumed that the awareness process either diffuses with a constant rate or follows an 
International Journal of Mathematical, Engineering and Management Sciences

Vol. 4, No. 3, 697-707, 2019

https://dx.doi.org/10.33889/IJMEMS.2019.4.3-055

exponential distribution function while the adoption process may take different functional forms. Following three distribution function are applied to express the eventual adoption process:

(i) Exponential distribution: It is relatively simple and commonly used distribution function for depicting the growth rate of new products. According to exponential distribution function, adoption occurs with a constant rate.

(ii) Logistic distribution: It depicts an S-shaped curve with scale and learning parameter. According to logistic distribution, adoption of new products increases over time via learning effect.

(iii) Normal distribution: It is a most important continuous distribution function having a capability to describe the adoption process based on different factors such as compatibility, complexity, etc. According to normal distribution, rate of adoption follows a perfect bellshape structure.

Corresponding to these distribution functions for the adoption process, Table 1 summarizes the mean value functions for different stochastic diffusion model.

Table 1. Proposed diffusion models

\begin{tabular}{|c|c|c|c|}
\hline Model & $\begin{array}{c}\text { Distribution } \\
\text { function }\left(F_{a w}(t)\right)\end{array}$ & $\begin{array}{c}\text { Distribution } \\
\text { function }\left(F_{a d}(t)\right)\end{array}$ & Mean value function $\bar{N}(t)$ \\
\hline $\begin{array}{c}\text { SIDM } \\
-1\end{array}$ & $t \sim$ const & $t \sim \operatorname{logistic}(b, \beta)$ & $m\left[1-\left(\frac{(1+\beta) e^{-b t}}{1+\beta e^{-b t}}\right) e^{\sigma^{2} t / 2}\right]$ \\
\hline $\begin{array}{c}\text { SIDM } \\
-2\end{array}$ & $t \sim \exp (b)$ & $t \sim \exp (b)$ & $\left.m\left[1-\left(1-\left(1-e^{-b t}\right)-(1+\beta) e^{-b t} \log \left(\frac{(1+\beta) e^{-b t}}{1+\beta e^{-b t}}\right)\right) e^{\sigma^{2} t / 2}\right] / 2\right]$ \\
\hline $\begin{array}{c}\text { SIDM }^{2} \\
-3\end{array}$ & $t \sim \exp (b)$ & $t \sim \operatorname{logistic}(b, \beta)$ & {$\left[1-\left(1-\varphi(t, \mu, s)+\varphi\left(t, \mu+b s^{2}, s\right) \exp \left(-b t+\mu b+\frac{(b s)^{2}}{2}\right)\right) e^{\sigma^{2} t / 2}\right]$} \\
\hline $\begin{array}{c}\text { SIDM } \\
-4\end{array}$ & $t \sim \exp (b)$ & $t \sim \operatorname{Normal}\left(\mu, s^{2}\right)$ & $m[1]$ \\
\hline
\end{tabular}

\section{An Empirical Study}

A case of Smartphone and automobile industries was taken to conduct the empirical analysis on the proposed modeling framework. The historical sales data of Apple iPhone (DS1) from the $3^{\text {rd }}$ quarter of 2007 to $1^{\text {st }}$ quarter of 2018 and annual data of Toyota Prius (DS2) from the period 2001 to 2014 were obtained from online source ${ }^{1}$ and published article (Lavasani et al., 2016) respectively. To estimate the parameters of the proposed models, a non-linear least square estimation procedure (Srinivasan and Mason, 1986) is employed using the SAS (SAS/ETS User's Guide, 2004) software package. The proposed models forecasting capabilities are tested by withholding the portion of the actual data set (withhold or testing data), comprising of the most recent observations. The remaining data set (training data) is used to estimate the parameters of the models and predict the values of testing data (Michalakelis et al., 2010). The testing sample is

${ }^{1}$ https://www.statista.com/statistics/263401/global-apple-iphone-sales-since-3rd-quarter-2007 (Accessed April. 26, 2018) 
International Journal of Mathematical, Engineering and Management Sciences

Vol. 4, No. 3, 697-707, 2019

https://dx.doi.org/10.33889/IJMEMS.2019.4.3-055

applied with 4 (for DS1) and 2 (for DS2) data points. Firstly, the parameter estimation is performed on the training data. For DS1 training sample is from $3^{\text {rd }}$ quarter of 2007 to $1^{\text {st }}$ quarter of 2017 and for DS2 data is taken is from 2001 to 2012. The results of the parameter estimation are listed in Table 2.

The statistical measures such as coefficient of determination $\left(R^{2}\right)$ and Root Mean Square Error (MSE) are calculated to measure the fitness of the proposed models. The results of the performance measures are summarized in Table 3. Further, the estimated parameters from the training data are used to forecast the cumulative adopters for the withhold samples. The forecasted values and actual values for the withhold sample is then compared and Mean Absolute Percentage Error (MAPE) is calculated. MAPE is an effective performance measure that validates forecasting accuracy of the proposed models. The values of MAPE for different SIDMs are listed in Table 3. From Table 3, it can be interpreted that all the developed diffusion models have a good ability to explain the demand variation in the sales data. Moreover, the value of MAPE for SIDM-1, SIDM-3 and SIDM-4 for both the dataset is $<10 \%$ and for SIDM-2 it lies between the interval [10\%, 20\%]. Therefore, it can be inferred that the forecasting ability of all the proposed models are highly accurate. Further, the goodness-of-fit curves for all the four models are depicted in Figure 1.

Table 2. Parameter estimation results

\begin{tabular}{|c|c|c|c|c|c|c|}
\hline Models & \multicolumn{7}{|c|}{ Parameters } \\
\hline & $\boldsymbol{m}$ & $\boldsymbol{b}$ & $\beta$ & $\sigma$ & \multicolumn{2}{c|}{$\boldsymbol{s}$} \\
\hline \multicolumn{7}{|c|}{ Apple iPhone (DS1) } \\
\hline SIDM-1 & 1448.97 & 0.153493 & 130.6439 & 0.005844 & - & - \\
\hline SIDM-2 & 40064.24 & 0.00635 & - & 0.006246 & - & - \\
\hline SIDM-3 & 1365.04 & 0.172456 & 78.55815 & 0.00652 & - & - \\
\hline SIDM-4 & 1742.36 & 0.285 & - & 0.0542 & 30.47356 & 12.63799 \\
\hline \multicolumn{7}{|c|}{ Toyota Prius $($ DS2) } \\
\hline SIDM-1 & 1775.04 & 0.299147 & 7.621 & 0.280216 & - & - \\
\hline SIDM-2 & 11285.6 & 0.05093 & - & 0.048776 & - & - \\
\hline SIDM-3 & 1620.22 & 0.419208 & 13.574 & 0.099497 & - & - \\
\hline SIDM-4 & 1494.35 & 0.38624 & - & 0.15632 & 5.775164 & 3.10052 \\
\hline
\end{tabular}

Table 3. Statistical measure results

\begin{tabular}{|l|c|c|c|c|c|c|}
\hline \multirow{2}{*}{ Models } & \multicolumn{5}{|c|}{ Performance Measures } \\
\hline & $\boldsymbol{R M S E}$ & $\boldsymbol{R}^{\mathbf{2}}$ & MAPE & $\boldsymbol{R M S E}$ & $\boldsymbol{R}^{2}$ & MAPE \\
\cline { 2 - 7 } & \multicolumn{7}{|c|}{ (training sample) } & (testing sample) & \multicolumn{3}{c|}{ Toyota Prius (DS2) } \\
\hline \multirow{2}{*}{ SIDM-1 } & 13.1792 & 0.9986 & $6.86 \%$ & 16.8149 & 0.9986 & $3.56 \%$ \\
\hline SIDM-2 & 51.5439 & 0.9783 & $15.76 \%$ & 34.8027 & 0.9942 & $15.86 \%$ \\
\hline SIDM-3 & 18.7457 & 0.9972 & $9.15 \%$ & 16.1817 & 0.9987 & $5.19 \%$ \\
\hline SIDM-4 & 8.6264 & 0.9994 & $1.27 \%$ & 14.6275 & 0.9990 & $0.96 \%$ \\
\hline
\end{tabular}


International Journal of Mathematical, Engineering and Management Sciences

Vol. 4, No. 3, 697-707, 2019

https://dx.doi.org/10.33889/IJMEMS.2019.4.3-055

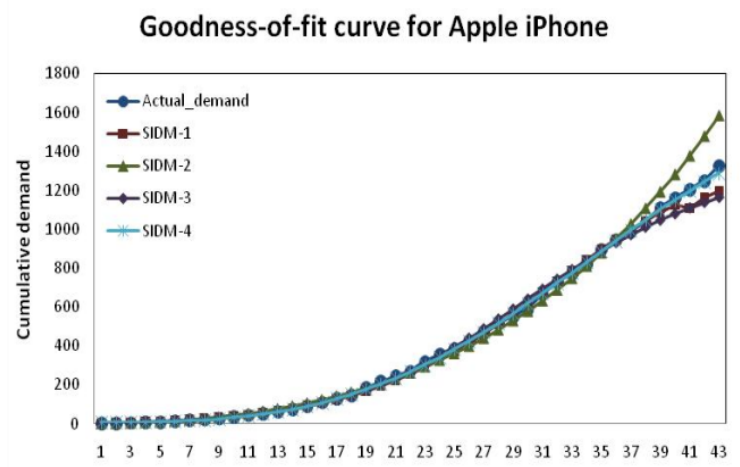

Goodness-of-fit curve for Toyota Prius

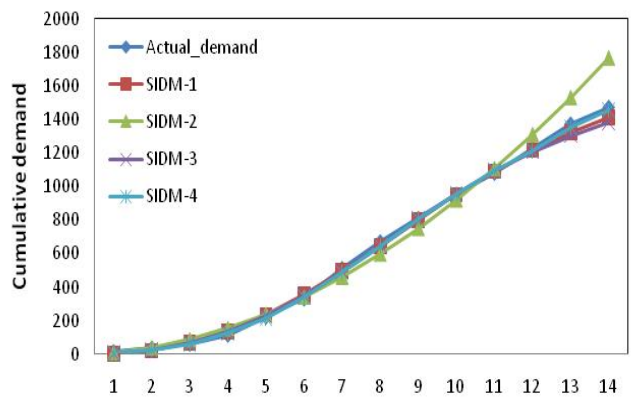

Figure 1. Goodness-of-fit curves for Apple iPhone (DS1) and Toyota Prius (DS2) datasets

\section{Concluding Remarks}

The stochastic differential equation based diffusion models are proposed in the present research to analyze the market penetration of Smartphone and automobile products. The study of market penetration pattern of technology products assists decision-makers and marketing managers in planning and expediting the policies pertaining to marketing, advertising, and production. The proposed diffusion models are formulated using a two-stage structure wherein first stage models the awareness of information process while the second stage represents the eventual adoption process. Convolution theory is employed to represent the awareness-adoption hazard rate function. The main purpose of the proposed study is to demonstrate the dissemination of new technology in a realistic environment by considering random fluctuation in the adoption rate. The forecasting capability of the developed models is examined by dividing the datasets into two portions: training data for fitting the models and testing data to analyse the forecasting accuracy. The proposed diffusion models contribute significantly in the field of technology forecasting and management science. Moreover, the results of the empirical analysis will provide essential suggestions for designing marketing strategy in Smartphone and automobile industries.

\section{Conflict of Interest}

The authors declare that there is no conflict of interest for this publication.

\section{Acknowledgment}

The research work presented in this paper is supported by grants to the second and third author from DST, via DST PURSE phase II, India.

\section{References}

Agarwal, M., Aggrawal, D., Anand, A., \& Singh, O. (2017). Modeling multi-generation Innovation Adoption based on conjoint effect of awareness process. International Journal of Mathematical, Engineering and Management Sciences, 2(2), 74-84. 
International Journal of Mathematical, Engineering and Management Sciences

Vol. 4, No. 3, 697-707, 2019

https://dx.doi.org/10.33889/IJMEMS.2019.4.3-055

Anand, A., \& Bansal, G. (2016). Predicting customer's satisfaction (dissatisfaction) using logistic regression. International Journal of Mathematical, Engineering and Management Sciences, 1(2), 77-88.

Anand, A., Kapur, P.K., Agarwal, M., \& Aggrawal, D. (2014, October). Generalized innovation diffusion modeling \& weighted criteria based ranking. In Reliability, Infocom Technologies and Optimization (ICRITO) (Trends and Future Directions), 2014 3rd International Conference on (pp. 1-6). IEEE.

Anand, A., Singhal, S., \& Singh, O. (2016, September). Multi-stage diffusion dynamics based on optimal control theory. In Reliability, Infocom Technologies and Optimization (Trends and Future Directions) (ICRITO), 2016 5th International Conference on (pp. 100-106). IEEE.

Anand, A., Singhal, S., \& Singh, O. (2018). Revisiting dynamic potential adopter diffusion models under the influence of irregular fluctuations in adoption rate. In Handbook of Research on Promoting Business Process Improvement Through Inventory Control Techniques (pp. 499-519). IGI Global.

Anand, A., Singhal, S., Panwar, S., \& Singh, O. (2018). Optimal price and warranty length for profit determination: an evaluation based on preventive maintenance. In Quality, IT and Business Operations (pp. 265-277). Springer, Singapore.

Aronov, I., \& Zazhigalkin, A. (2017). Diffusion of innovations: patenting or standardization. International Journal of Mathematical, Engineering and Management Sciences, 2(2), 64-73

Bass, F.M. (1969). A new product growth for model consumer durables. Management science, 15(5), 215227.

Boswijk, H.P., \& Franses, P.H. (2005). On the econometrics of the Bass diffusion model. Journal of Business \& Economic Statistics, 23(3), 255-268.

Chandrasekaran, D., \& Tellis, G.J. (2007). A critical review of marketing research on diffusion of new products. In Review of Marketing research (pp. 39-80). Emerald Group Publishing Limited

Eliashberg, J., \& Chatterjee, R. (1986). Stochastic issues in innovation diffusion models. In Innovation Diffusion Models of New Product. In Vijay Mahajan \& Yoram Wind (Eds), Cambridge, MA: Ballinger Publishing Company, 151-203.

Fanelli, V., \& Maddalena, L. (2012). A time delay model for the diffusion of a new technology. Nonlinear Analysis: Real World Applications, 13(2), 643-649.

Giovanis, A.N., \& Skiadas, C.H. (1999). A stochastic logistic innovation diffusion model studying the electricity consumption in Greece and the United States. Technological Forecasting and Social Change, 61(3), 235-246.

Gutiérrez, R., Nafidi, A., \& Sánchez, R. G. (2005). Forecasting total natural-gas consumption in Spain by using the stochastic Gompertz innovation diffusion model. Applied Energy, 80(2), 115-124.

Kalish, S. (1985). A new product adoption model with price, advertising, and uncertainty. Management science, 31(12), 1569-1585.

Lal, V.B., \& Kaicker, S. (1988). Modeling innovation diffusion with distributed time lag. Technological Forecasting and Social Change, 34(2), 103-113.

Landsman, V., \& Givon, M. (2010). The diffusion of a new service: combining service consideration and brand choice. $Q M E, 8(1), 91-121$.

Lavasani, M., Jin, X., \& Du, Y. (2016). Market penetration model for autonomous vehicles on the basis of earlier technology adoption experience. Transportation Research Record, 2597(1), 67-74.

Meade, N., \& Islam, T. (2006). Modelling and forecasting the diffusion of innovation-A 25-year review. International Journal of forecasting, 22(3), 519-545. 
International Journal of Mathematical, Engineering and Management Sciences

Vol. 4, No. 3, 697-707, 2019

https://dx.doi.org/10.33889/IJMEMS.2019.4.3-055

Michalakelis, C., Varoutas, D., \& Sphicopoulos, T. (2010). Innovation diffusion with generation substitution effects. Technological Forecasting and Social Change, 77(4), 541-557.

Negahban, A., \& Smith, J.S. (2014). Production-sales policies for new product diffusion under stochastic supply. In Proceedings of the Sixth International Conference on Advances in System Simulation (pp. 197-202). Red Hook, NY: International Academy, Research, and Industry Association (IARIA).

$\emptyset \mathrm{ksendal}$, B. (2003). Stochastic differential equations. In Stochastic differential equations (pp. 65-84). Berlin Heidelberg: Springer.

Rogers E. (2003). The Diffusion of innovations. Fifth edition. The Free Press, New York

SAS Institute Inc. (2004). SAS/ETS User's Guide Version 9.1, SAS Institute Inc., Cary, NC.

Singh, O., Anand, A., Kapur, P.K., \& Aggrawal, D. (2012). Consumer behaviour-based innovation diffusion modelling using stochastic differential equation incorporating change in adoption rate. International Journal of Technology Marketing, 7(4), 346-360.

Singhal, S., Anand, A., \& Singh, O. (2019). Understanding multi-stage diffusion process in presence of attrition of potential market and related pricing policy. Yugoslav Journal of Operations Research, https://doi.org/10.2298/YJOR1803.

Srinivasan, V., \& Mason, C.H. (1986). Nonlinear least squares estimation of new product diffusion models. Marketing Science, 5(2), 169-178.

Wang, W., Fergola, P., Lombardo, S., \& Mulone, G. (2006). Mathematical models of innovation diffusion with stage structure. Applied Mathematical Modelling, 30(1), 129-146.

Wang, Y., Pei, L., \& Wang, Z. (2017). The NLS-based grey Bass model for simulating new product diffusion. International Journal of Market Research, 59(5), 655-669. 